\title{
CIAPIN1 gene silencing enhances chemosensitivity in a drug-resistant animal model in vivo
}

\author{
X.M. Wang ${ }^{1}$, S.J. Gao ${ }^{1}$, X.F. Guo ${ }^{1}$, W.J. Sun ${ }^{1}$, Z.Q. Yan ${ }^{2}$, W.X. Wang ${ }^{1}$, Y.Q. Xu ${ }^{1}$ and D. Lu ${ }^{1}$ \\ ${ }^{1}$ Department of Oncology, The Second Affiliated Hospital, Harbin Medical University, Harbin, China \\ ${ }^{2}$ Department of Breast Surgery, The Second Affiliated Hospital, Harbin Medical University, Harbin, China
}

\begin{abstract}
Overexpression of cytokine-induced apoptosis inhibitor 1 (CIAPIN1) contributes to multidrug resistance (MDR) in breast cancer. This study aimed to evaluate the potential of CIAPIN1 gene silencing by RNA interference (RNAi) as a treatment for drug-resistant breast cancer and to investigate the effect of CIAPIN1 on the drug resistance of breast cancer in vivo. We used lentivirus-vector-based RNAi to knock down CIAPIN1 in nude mice bearing MDR breast cancer tumors and found that lentivirus-vector-mediated silencing of CIAPIN1 could efficiently and significantly inhibit tumor growth when combined with chemotherapy in vivo. Furthermore, Western blot analysis showed that both CIAPIN1 and P-glycoprotein expression were efficiently downregulated, and P53 was upregulated, after RNAi. Therefore, we concluded that lentivirus-vector-mediated RNAi targeting of CIAPIN1 is a potential approach to reverse MDR of breast cancer. In addition, CIAPIN1 may participate in MDR of breast cancer by regulating P-glycoprotein and P53 expression.
\end{abstract}

Key words: CIAPIN1 gene; Multidrug resistance; RNA interference; MDR1 gene; Breast neoplasms

\section{Introduction}

Breast cancer is the most common cancer of women worldwide, accounting for $22.9 \%$ of all female cancers. In China, the prevalence of breast cancer has been increasing (1). The predominant cause of death in breast cancer patients is distant metastasis, and chemoradiotherapy is the most effective approach against advanced breast cancer $(2,3)$. Because chemotherapy is so important as a breast cancer treatment, multidrug resistance (MDR) can be a major obstacle (4). MDR is a complicated process that involves many mechanisms, one of which is the overexpression of energy-dependent transporters of anti-cancer drugs into and out of cells $(5,6)$. The transporter P-glycoprotein (P-gp or MDR1), a product of the MDR1 gene, is responsible for resistance to doxorubicin and taxanes in cultured cells (7), and plays a key role in breast cancer MDR. Studies on blocking or competitively inhibiting P-gp have been carried out, but the use of many agents, including the calcium channel blocker verapamil and the immunosuppressive agent cyclosporine, is restricted by their dose-limiting toxicity (6). Therefore, it is necessary to develop new approaches inhibiting the expression of P-gp to overcome MDR in breast cancer (8).

Cytokine-induced apoptosis inhibitor 1 (CIAPIN1) is a newly identified anti-apoptotic protein that has no homology with Bcl-2, caspase, and IAP families or signal-transduction molecules that regulate apoptosis (9). CIAPIN1 is associated with MDR in SGC7901/Adr gastric cancer cells $(10,11)$ and HL-60/Adr leukemia cells (12) in which CIAPIN1 confers resistance by upregulating MDR1 expression (10-12). Recently, we reported the same MDR mechanism in MCF-7/ADM breast cancer cells (13). When the expression of CIAPIN1 was downregulated by RNA interference (RNAi) in MCF-7/ADM breast cancer cells, drug resistance was significantly reduced by inhibiting MDR1 expression (13). Thus, the CIAPIN1 gene is assumed to be a potential target in treating breast cancer MDR $(13,14)$. In this study, we evaluated CIAPIN1-RNAi as a potential therapy for drug-resistant breast cancer. This is the first report of CIAPIN1 and reversal of MDR of breast cancer in vivo.

Correspondence: D. Lu, Department of Oncology, The Second Affiliated Hospital, Harbin Medical University, Harbin 150086, China. E-mail: Iudan1972@medmail.com.cn 


\section{Material and Methods}

\section{CIAPIN1 short hairpin RNA (shRNA) lentivirus vector construction and production}

The CIAPIN1 shRNAs were designed and synthesized as previously described (13). Although three shRNAs were designed, the most efficient interfering sequence, which was determined in a previous study, was used here. The CIAPIN1 shRNA sequence was 5'-GATCCGGAGCC AGTAGAGACAGCTCTTCCTGTCAGAAGCTGTCTCTAC TGGCTCCTTTTTG-3'. The recombined plasmid of shRNAs targeting CIAPIN1 was synthesized as recently described (13). Lentivirus Packaging Plasmid Mix (System Biosciences, USA) and CIAPIN1-shRNA were cotransfected into 293TN cells (System Biosciences) to package and produce a lentiviral vector, and the viral titer was determined by the gradient dilution method. The final vital titer was adjusted to $5 \times 10^{5}$ infectious units (IFU)/ $\mu \mathrm{L}$ using Dulbecco's phosphate-buffered saline (DPBS), pH 7.8, and prepared for transfection.

\section{Cell lines, animal model, and chemotherapy- sensitivity assay}

All experiments were carried out according to the China Guide for the Care and Use of Experimental Animals. Research was approved by the Institute Committee of Animal Care of Harbin Medical University.

The multidrug-resistant human breast carcinoma cell line MCF-7/ADM and the parental, sensitive MCF-7 cell line were obtained from Shanghai Jiao Tong University and the CAS cell storeroom (China), respectively. MCF-7 and MCF-7/ADM cells were cultured in DMEM (Invitrogen, USA) in a $5 \% \mathrm{CO}_{2}$ incubator at $37^{\circ} \mathrm{C}$, supplemented with $10 \%$ fetal bovine serum (Gibco, USA) and antibiotics. Female BALB/C-nude mice (Shanghai Super-B\&K Laboratory Animal Corp., Ltd., China) weighing 18-20 g were used for tumor inoculation. The MDR breast cancer cells MCF-7/ADM in exponential growth phase were collected, and resuspended in fetal calf serum. Cell number and viability were determined by staining a small volume of cell suspension with a $0.2 \%$ trypan blue saline solution and examining the cells in a hemocytometer. The MCF-7/ADM cells $\left(1 \times 10^{6} /\right.$ mouse) were subcutaneously injected into the left armpit of each nude mouse. Fifteen days after inoculation, the implanted tumors became palpable, and the experimental treatments were initiated.

CIAPIN1-shRNA lentivirus vectors (Lv-CIAPIN1shRNA) were administered by caudal vein injection. The xenograft model mice were randomly divided into three groups of 10 mice each. In the RNAi-ADM group (group A), mice were given $50 \mu \mathrm{L}$ Lv-CIAPIN1-shRNA (lentivirus titer of $5 \times 10^{5} \mathrm{IFU} / \mu \mathrm{L}$ ) twice a day on days 1,4 , and 7 . They were then given doxorubicin $(1 \mu \mathrm{g} / \mu \mathrm{L}$; Pharmacia, Italy) diluted with normal saline once a day for 5 days at a dose of $5 \mathrm{mg} / \mathrm{kg}$. In the ADM group (group B), mice were given $50 \mu \mathrm{L}$ normal saline twice a day on days 1,4 , and 7 .
They were then given doxorubicin $(1 \mu \mathrm{g} / \mu \mathrm{L})$ diluted with normal saline once a day for 5 days at a dose of $5 \mathrm{mg} / \mathrm{kg}$. In the control group (group C), mice were injected with normal saline following the same schedules as the experimental animals. Animals were killed 10 days after the last doxorubicin or saline injection.

The sensitivity of breast cancer tumors to doxorubicin was measured by inhibition of the rate of increase in tumor weight calculated as: Inhibition rate $=(1$-average tumor weight of experimental group/average tumor weight of control group) $\times 100 \%$.

\section{Western blot analysis}

Tissue samples were split, and protein extraction was conducted with Tissue Protein Extraction Reagent (T-PER, 78510, Pierce, USA) in accordance with the instruction manual. Briefly, tissue samples were washed twice with DPBS and then were mixed with T-PER at a ratio of 1:20. The lysates were centrifuged at $10,000 \mathrm{~g}$ for $5 \mathrm{~min}$ and supernatants were collected. The concentration of total protein in the supernatant was measured by bicinchoninic acid assay and adjusted to a concentration of $10 \mu \mathrm{g} / \mu \mathrm{L}$. Immunoblotting was conducted as previously described (13). The following antibodies were used: anti-CIAPIN1 (1:500, Abcam, UK), anti-P53 (1:300, Santa Cruz, USA), anti-P-gp (1:100, Abcam), anti- $\beta$-actin (1:1000, Santa Cruz), anti-mouse horseradish peroxidase (HRP)-conjugated antibody (1:5000, Santa Cruz) and anti-rabbit HRP-conjugated antibody (1:3000, Cell Signaling Technology, USA).

\section{Statistical analysis}

Western blot analyses were performed at least three times. Statistical analysis was performed using the twotailed Student $t$-test, and statistical significance is reported as $\mathrm{P}<0.05$.

\section{Results}

\section{CIAPIN1 shRNA enhanced the in vivo sensitivity to doxorubicin}

To investigate whether CIAPIN1 RNAi was capable of reducing drug resistance in vivo, the effects of doxorubicin chemotherapy alone or in combination with CIAPIN1 shRNA on the growth of drug-resistant breast cancer xenografts were evaluated in transplanted MCF-7/ADM tumors. Fifteen days after tumor cell inoculation, 30 tumor-bearing mice were randomized into three groups for treatment. All mice were killed on day 22. The inhibition rates in groups A and B were $50.1 \pm 8.77 \%$ and $4.36 \pm 6.27 \%$, respectively. As expected, the inhibition rate was significantly greater in group $A$ than in group $B(P<0.05)$. However, no difference of inhibition rate was observed between groups $B$ and $C$, which may have resulted from the drug resistance of MCF-7/ADM cells $(P>0.05$; Figure 1). Obviously, group $A$ had stronger anticancer effects compared with the other two groups, which 


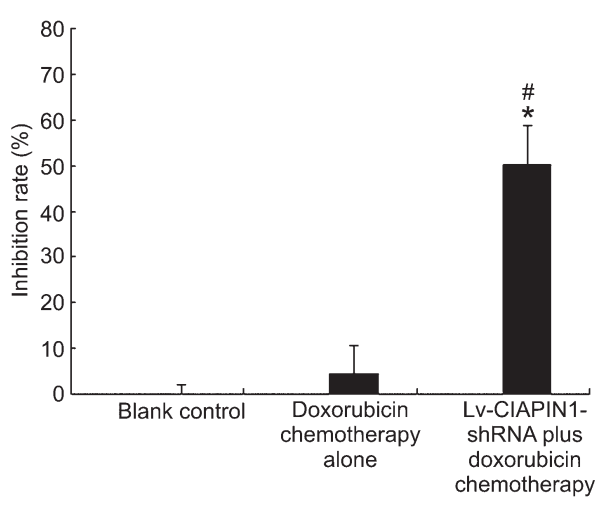

Figure 1. Lv-CIAPIN1-shRNA plus doxorubicin chemotherapy significantly increased the tumor inhibition rate. ${ }^{*} \mathrm{P}<0.01 \mathrm{com}-$ pared with doxorubicin chemotherapy group; ${ }^{\#} \mathrm{P}<0.01$ compared with blank control ( $t$-test).

led to the conclusion that Lv-CIAPIN1-shRNA enhanced the sensitivity of tumor cells to doxorubicin in drugresistant breast cancer xenografts in this nude mouse model.

\section{CIAPIN1 shRNA inhibited CIAPIN1 expression in transplanted tumor cells}

We measured CIAPIN1 expression to evaluate the effectiveness of LV-CIAPIN1-shRNA on silencing CIAPIN1 in vivo and to explore the relationship between CIAPIN1 expression and breast cancer drug resistance. The levels of the CIAPIN1 protein in groups $A, B$, and $C$ were $0.277 \pm 0.075,0.823 \pm 0.138$, and $0.828 \pm 0.133$, respectively. The levels of the CIAPIN1 protein in group A were significantly lower $(P<0.05)$ than in the other two groups (Figures 2 and $3 \mathrm{~A}$ ). These results suggested that CIAPIN1 shRNA inhibited CIAPIN1 expression, which was in accordance with the sensitivity to doxorubicin.

\section{CIAPIN1 shRNA downregulated P-gp expression}

To investigate the effect of CIAPIN1 on MDR1 expression of breast cancer in vivo, P-gp, which was encoded by MDR1, was determined by Western blotting.

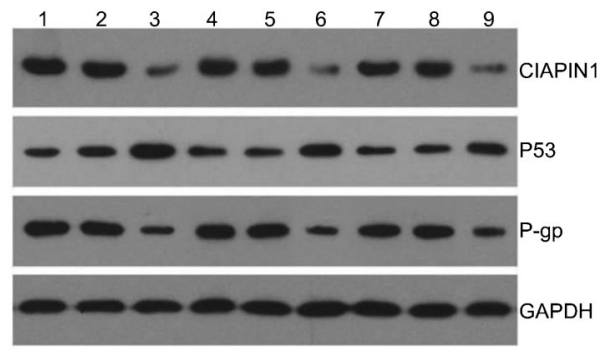

Figure 2. Expression of CIAPIN1, P53, and P-gp protein in each group by Western blot. Lanes 1, 4, 7, blank control; lanes 2, 5, 8, ADM group; lanes 3, 6, 9: RNAi-ADM group.

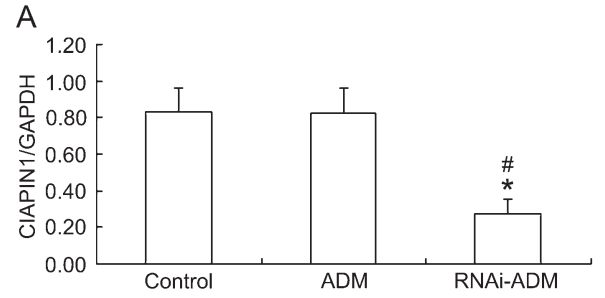

B

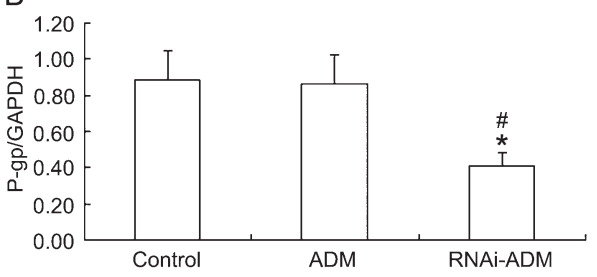

C

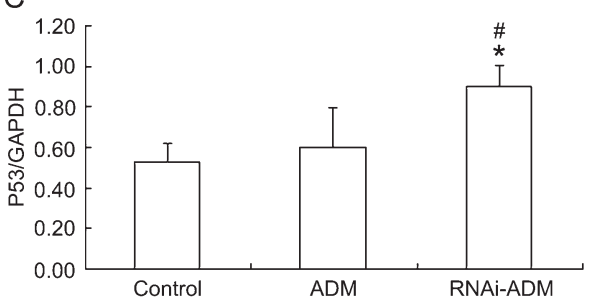

Figure 3. Expression of CIAPIN1 protein (A), P-gp protein (B), and $\mathrm{P} 53$ protein $(\mathrm{C})$ in inoculated tumor. ${ }^{*} \mathrm{P}<0.05$ compared with blank control; ${ }^{*} \mathrm{P}<0.05$ compared with doxorubicin chemotherapy group (ADM) ( $t$-test).

The levels of the P-gp protein in groups $A, B$, and $C$ were $0.410 \pm 0.070,0.863 \pm 0.158$, and $0.884 \pm 0.163$, respectively. As expected, the expression of P-gp in group A was significantly lower $(P<0.05)$ that in the other two groups (Figures 2 and $3 \mathrm{~B}$ ). The results indicated that CIAPIN1 may reverse drug resistance to doxorubicin in model animals by inhibiting P-gp expression.

\section{CIAPIN1 elevated P53 expression}

The levels of the P53 protein, measured by Western blotting, were $0.901 \pm 0.104$ in group $A, 0.598 \pm 0.196$ in group $B$, and $0.525 \pm 0.094$ in group $C$. The expression of $P 53$ in group $A$ was thus significantly greater $(P<0.05)$ than in the other two groups (Figures 2 and $3 C$ ). These results indicated that CIAPIN1 may participate in drug resistance of breast cancer by regulating P53 expression.

\section{Discussion}

As mentioned above, MDR remains a dominant impediment to curative breast cancer chemotherapy. Over $90 \%$ of metastatic breast cancer patients experience failure of breast cancer treatment due to MDR (15). Therefore, it is urgent to develop new approaches to 
overcome MDR of breast cancer. Many genes and mechanisms are involved in MDR of breast cancer. While gene therapy has become a focus of cancer research, the current study aims to improve the efficacy of chemotherapy by targeting the genes related to breast cancer MDR. CIAPIN1, a novel apoptosis inhibitor, was first identified in 2004 by Shibayama et al. (9). In recent years, evidence that CIAPIN1 takes part in the MDR process of malignant cancers was obtained from studies of MDR in gastric cancer and leukemia cell lines (10-12). Our previous investigation of MDR in human breast cancer cells arrived at the same conclusion (13). Therefore, CIAPIN1 is a potential target to improve therapeutic effects.

Since CIAPIN1 expression is frequently elevated in MDR breast cancer, we used RNAi to downregulate CIAPIN1 expression in this study. RNAi is an evolutionarily conserved, posttranscriptional gene-silencing mechanism in which small interfering 21-23 mer doublestranded RNA (siRNA) mediates sequence-specific degradation of mRNA (16). RNAi-based gene therapies have been successfully conducted in a variety of disease models (17). Compared to RNAi in vitro, RNAi in vivo requires high-efficiency, sustainability, and safety. It is known that naked siRNA or even lipid-complexed siRNA has a short half-life in cell plasmids and is cleared rapidly through the liver and kidneys, resulting in inefficient and transient gene silencing (18). shRNAs can be expressed intracellularly from plasmid DNA. Once delivered with vectors, the continuous endogenous expression of shRNAs would lead to more efficient and longer-lasting gene silencing (19). Therefore, we applied shRNAs in the present study. In addition, incorporating siRNA with particular carriers appears to be an efficient approach to achieve sustained gene silencing in vivo (17-19). As lentivirus can be integrated into the host genome and demonstrates long-term expression of integrated genes, and because lentivirus has almost no immunogenicity, a lentivirus vector was selected in this study (19-21). Our study adopted the third-generation lentiviral vector that had the least relativity with wild-type human HIV-1 virus, and this improved the safety of the vector dramatically. Our previous study showed that lentivirus could deliver CIAPIN1-shRNA into MCF-7/ADM cells with high efficiency (13).

The Lv-CIAPIN1-shRNAs were delivered to the animals by caudal vein injection, and followed by chemotherapy. At the end of this study, we evaluated the effect of RNAi targeting CIAPIN1 on MDR by measuring the inhibition of increase in tumor weight. Results of the current study showed that the anti-tumor effect mediated by LV-CIAPIN1-shRNA in combination with doxorubicin was significantly improved, which may be due to successful delivery of CIAPIN1 shRNAs to MDR breast cancer cells in vivo. The CIAPIN1 shRNAs downregulated CIAPIN1 expression in MDR breast cancer cells and improved the effect of chemotherapy on MDR breast cancer cells, which led to the inhibition of tumor growth. In contrast, there was hardly any difference between doxorubicin alone and the blank controls, because the cells inoculated into the nude mice were resistant to doxorubicin. The results demonstrated that lentivirusvector-mediated RNAi targeting CIAPIN1 enhanced the chemosensitivity of breast cancer in vivo. Additionally, no mice died in the current study, which supports the safety of RNAi targeting of CIAPIN1 in vivo. Furthermore, we detected expression of CIAPIN1 at the protein level by Western blot after the mice were killed. CIAPIN1 protein expression in group A was significantly lower compared with the other two groups, indicating that Lv-CIAPIN1shRNAs integrated into the genome of MDR breast cancer cells and led to CIAPIN1 gene silencing. The results revealed that LV-CIAPIN1-shRNAs can downregulate the expression of CIAPIN1 not only in cultured breast cancer cells but also in tumor tissue. It strongly suggests that RNAi targeting of CIAPIN1 is a potential method to improve the efficacy of chemotherapy in the clinic. As the clinical safety of the viral vector has not been confirmed, further research is needed to establish an efficient gene delivery system such as chitosan, polymer, or other nanomaterials. In order to improve a more effective targeting therapy, two approaches can be taken, one to develop a new vector with targeting delivery as described above, and another to look for an RNAi-specific expression promoter. The former may enrich vector DNA complexes in specific cells, and the latter may induce gene expression in specific cells. In addition, because human full genome sequence information is incomplete, when choosing siRNA sequences, only limited sequence information can be compared, which may result in offtarget application. Therefore, this condition exists theoretically but presents problems that may frustrate progress in optimizing the sequence of siRNA.

As described previously, overexpression of P-gp on the tumor cell membrane is the most important mechanism of MDR in breast cancer, especially P-gp encoded by MDR1. Our study demonstrated that the expression of P-gp was inhibited when CIAPIN1 was downregulated in tumor tissue. Downregulation of P-gp elevated the accumulation of intracellular drugs and improved the effect of drugs on tumor tissue. The results validated CIAPIN1 participation in MDR of breast cancer by regulating P-gp.

P53 plays a vital role in carcinogenesis, and many studies have revealed that it is involved with MDR (22). In addition to regulation of apoptosis, P53 modulates the expression of P-gp. Several studies have reported that wild-type P53 represses expression of the MDR1 gene $(23,24)$ and mutant P53 exerts a stimulatory effect $(23,25)$. The current study showed that P-gp expression was suppressed while wild-type P53 expression was upregulated when CIAPIN1 expression was downregulated. Since MCF-7 cells harbor wild-type P53 (26), we assumed that the CIAPIN1 gene may upregulate P-gp expression by 
regulating wild-type P53 expression, leading to breast cancer resistance. However, there are controversies about the modulation of P-gp expression by P53 in the different cancer cell lines that have been studied (23-26). It must be emphasized that this study was performed using only MCF-7/ADM cells, and whether this conclusion still holds for other breast cancer cell lines still needs further study.

In conclusion, CIAPIN1 is involved in the development of breast cancer MDR by modulating P-gp and P53. Lentivirus-mediated CIAPIN1 silencing could reinstate the chemotherapy sensitivity of MDR breast cancer in vivo.

\section{References}

1. Guo P, Huang ZL, Yu P, Li K. Trends in cancer mortality in China: an update. Ann Oncol 2012; 23: 2755-2762, doi: 10.1093/annonc/mds069.

2. Seruga B, Hertz PC, Le LW, Tannock IF. Global drug development in cancer: a cross-sectional study of clinical trial registries. Ann Oncol 2010; 21: 895-900, doi: 10.1093/ annonc/mdp403.

3. Marquette C, Nabell L. Chemotherapy-resistant metastatic breast cancer. Curr Treat Options Oncol 2012; 13: 263-275, doi: 10.1007/s11864-012-0184-6.

4. Gonzalez-Angulo AM, Morales-Vasquez F, Hortobagyi GN. Overview of resistance to systemic therapy in patients with breast cancer. Adv Exp Med Biol 2007; 608: 1-22, doi: 10.1007/978-0-387-74039-3_1.

5. Kuo MT. Roles of multidrug resistance genes in breast cancer chemoresistance. Adv Exp Med Biol 2007; 608: 2330, doi: 10.1007/978-0-387-74039-3_2.

6. Cascorbi I. Role of pharmacogenetics of ATP-binding cassette transporters in the pharmacokinetics of drugs. Pharmacol Ther 2006; 112: 457-473, doi: 10.1016/j.pharmthera.2006.04. 009.

7. Deng L, Tatebe S, Lin-Lee YC, Ishikawa T, Kuo MT. MDR and MRP gene families as cellular determinant factors for resistance to clinical anticancer agents. Cancer Treat Res 2002; 112: 49-66, doi: 10.1007/978-1-4615-1173-1_3.

8. Li YT, Chua MJ, Kunnath AP, Chowdhury EH. Reversing multidrug resistance in breast cancer cells by silencing $A B C$ transporter genes with nanoparticle-facilitated delivery of target siRNAs. Int $J$ Nanomedicine 2012; 7: 24732481.

9. Shibayama H, Takai E, Matsumura I, Kouno M, Morii E, Kitamura $\mathrm{Y}$, et al. Identification of a cytokine-induced antiapoptotic molecule anamorsin essential for definitive hematopoiesis. J Exp Med 2004; 199: 581-592, doi: 10.1084/jem.20031858.

10. Hao Z, Li X, Qiao T, Du R, Hong L, Fan D. CIAPIN1 confers multidrug resistance by upregulating the expression of MDR-1 and MRP-1 in gastric cancer cells. Cancer Biol Ther 2006; 5: 261-266, doi: 10.4161/cbt.5.3.2381.

11. Li X, Fan R, Zou X, Hong L, Gao L, Jin H, et al. [Reversal of multidrug resistance of gastric cancer cells by downregulation of CIAPIN1 with CIAPIN1 siRNA]. Mol Biol 2008; 42: 102-109, doi: 10.1016/j.jmb.2008.05.062.

12. Li $X$, Hong $L$, Zhao $Y$, Jin $H$, Fan R, Du R, et al. A new apoptosis inhibitor, CIAPIN1 (cytokine-induced apoptosis
Therefore, stable CIAPIN1 RNAi holds great promise as a new approach to reverse breast cancer MDR. Further research is needed of the dose dependency of therapeutic effects on RNAi and doxorubicin, and the effect of CIAPIN1 silencing on organ function and blood chemistry.

\section{Acknowledgments}

Research supported by the Science and Technology Foundation provided by the Education Department of Heilongjiang Province (\#12521245). inhibitor 1), mediates multidrug resistance in leukemia cells by regulating MDR-1, Bcl-2, and Bax. Biochem Cell Biol 2007; 85: 741-750, doi: 10.1139/O07-141.

13. Lu D, Xiao Z, Wang W, Xu Y, Gao S, Deng L, et al. Down regulation of CIAPIN1 reverses multidrug resistance in human breast cancer cells by inhibiting MDR-1. Molecules 2012; 17: 7595-7611, doi: 10.3390/molecules17067595.

14. Li X, Wu K, Fan D. CIAPIN1 as a therapeutic target in cancer. Expert Opin Ther Targets 2010; 14: 603-610, doi: $10.1517 / 14728221003774127$

15. Egerton N. Ixabepilone (ixempra), a therapeutic option for locally advanced or metastatic breast cancer. P T 2008; 33 : 523-531.

16. Meister G, Tuschl T. Mechanisms of gene silencing by double-stranded RNA. Nature 2004; 431: 343-349, doi: 10.1038/nature02873.

17. Couto LB, High KA. Viral vector-mediated RNA interference. Curr Opin Pharmacol 2010; 10: 534-542, doi: 10.1016/ j.coph.2010.06.007.

18. Wang X, Chen $Y$, Ren J, Qu X. Small interfering RNA for effective cancer therapies. Mini Rev Med Chem 2011; 11 114-124, doi: 10.2174/138955711794519528.

19. Manjunath $\mathrm{N}, \mathrm{Wu} \mathrm{H}$, Subramanya S, Shankar P. Lentiviral delivery of short hairpin RNAs. Adv Drug Deliv Rev 2009; 61: 732-745, doi: 10.1016/j.addr.2009.03.004.

20. Jiang $R$, Xue $S$, Jin Z. Stable knockdown of MYCN by lentivirus-based RNAi inhibits human neuroblastoma cells growth in vitro and in vivo. Biochem Biophys Res Commun 2011; 410: 364-370, doi: 10.1016/j.bbrc.2011.06.020.

21. Santhosh CV, Tamhane MC, Kamat RH, Patel VV, Mukhopadhyaya R. A lentiviral vector with novel multiple cloning sites: stable transgene expression in vitro and in vivo. Biochem Biophys Res Commun 2008; 371: 546-550, doi: 10.1016/j.bbrc.2008.04.106.

22. Wei J, Zaika E, Zaika A. p53 Family: role of protein isoforms in human cancer. J Nucleic Acids 2012; 2012: 687359, doi: 10.1155/2012/687359.

23. Chin KV, Ueda K, Pastan I, Gottesman MM. Modulation of activity of the promoter of the human MDR-1 gene by Ras and p53. Science 1992; 255: 459-462, doi: 10.1126/ science. 1346476 .

24. Zhou G, Kuo MT. Wild-type p53-mediated induction of rat MDR-1b expression by the anticancer drug daunorubicin J Biol Chem 1998; 273: 15387-15394, doi: 10.1074/jbc.273. 25.15387. 
25. Lin J, Teresky AK, Levine AJ. Two critical hydrophobic amino acids in the $\mathrm{N}$-terminal domain of the $\mathrm{p} 53$ protein are required for the gain of function phenotypes of human p53 mutants. Oncogene 1995; 10: 2387-2390.
26. Zhang YF, Li XH, Shi YQ, Wu YY, Li N, He Q, et al. CIAPIN1 confers multidrug resistance through up-regulation of MDR1 and Bcl-L in LoVo/Adr cells and is independent of p53. Oncol Rep 2011; 25: 1091-1098. 